\title{
RANDOM ITERATION WITH PLACE DEPENDENT PROBABILITIES
}

\author{
BY
}

RAFAŁ KA PICA (Kraków) AND MACIEJ ŚLECZKA (Katowice)

\begin{abstract}
We consider Markov chains arising from random iteration of functions $S_{\theta}: X \rightarrow X, \theta \in \Theta$, where $X$ is a Polish space and $\Theta$ is an arbitrary set of indices. At $x \in X, \theta$ is sampled from a distribution $\vartheta_{x}$ on $\Theta$, and the $\vartheta_{x}$ are different for different $x$. Exponential convergence to a unique invariant measure is proved. This result is applied to the case of random affine transformations on $\mathbb{R}^{d}$, giving the existence of exponentially attractive perpetuities with place dependent probabilities.
\end{abstract}

2020 Mathematics Subject Classification: Primary 60J05; Secondary $37 \mathrm{~A} 25$.

Key words and phrases: random iteration of functions, exponential convergence, invariant measure, perpetuities.

\section{INTRODUCTION}

We consider the Markov chain of the form $X_{0}=x_{0}, X_{1}=S_{\theta_{0}}\left(x_{0}\right), X_{2}=$ $S_{\theta_{1}} \circ S_{\theta_{0}}\left(x_{0}\right)$ and inductively

$$
X_{n+1}=S_{\theta_{n}}\left(X_{n}\right),
$$

where $S_{\theta_{0}}, S_{\theta_{1}}, \ldots, S_{\theta_{n}}$ are randomly chosen from a family $\left\{S_{\theta}: \theta \in \Theta\right\}$ of functions that map a separable metric space $X$ into itself. If the chain starts at $x \in X$ then $\theta \in \Theta$ is sampled from a distribution $\vartheta_{x}$ on $\Theta$, where the $\vartheta_{x}$ are different for different $x$. The chain $\left(X_{n}\right)_{n \in \mathbb{N}_{0}}$ is defined in a rigorous way in Section 3. We are interested in the rate of convergence to a stationary distribution $\mu_{*}$ on $X$, i.e.

$$
P\left\{X_{n} \in A\right\} \rightarrow \mu_{*}(A) \quad \text { as } n \rightarrow \infty .
$$

If the above convergence is exponential then $\left(X_{n}\right)_{n \in \mathbb{N}_{0}}$ is called exponentially ergodic. In the case of constant probabilities, i.e. $\vartheta_{x}=\vartheta_{y}$ for $x, y \in X$, the basic tool when studying asymptotics of (1.1) is backward iterations $Z_{n+1}=$ $S_{\theta_{0}} \circ S_{\theta_{1}} \circ \cdots \circ S_{\theta_{n}}\left(x_{0}\right)$. Since $X_{n}$ and $Z_{n}$ are identically distributed and, under suitable conditions, $Z_{n}$ converges almost surely at exponential rate to some 
random element $Z$, one obtains exponential ergodicity of $\left(X_{n}\right)_{n \in \mathbb{N}_{0}}$ (see [6]). For place dependent $\vartheta_{x}$ we need a different approach because the distributions of $X_{n}$ and $Z_{n}$ are not equal.

The simplest case when $\Theta=\{1, \ldots, n\}$ is treated in [2] and [31], where the existence of a unique attractive invariant measure is established. A similar result holds true when $\Theta=[0, T]$ and $\vartheta_{x}$ are absolutely continuous (see [15]). Recently it was shown that the rate of convergence in the case of $\Theta=\{1, \ldots, n\}$ is exponential (see [28]).

In this paper we treat the general case of place dependent $\vartheta_{x}$ for arbitrary $\Theta$ and prove the existence of a unique exponentially attractive invariant measure for (1.1). Our approach is based on the coupling method which can be briefly described as follows. For arbitrary starting points $x_{0}, \bar{x}_{0} \in X$ we consider chains $\left(X_{n}\right)_{n \in \mathbb{N}_{0}}$, $\left(\bar{X}_{n}\right)_{n \in \mathbb{N}_{0}}$ with $X_{0}=x_{0}, \bar{X}_{0}=\bar{x}_{0}$ and try to build correlations between $\left(X_{n}\right)_{n \in \mathbb{N}_{0}}$ and $\left(\bar{X}_{n}\right)_{n \in \mathbb{N}_{0}}$ in order to make their trajectories as close as possible. This can be done since the transition probability function $\mathbf{B}_{x, y}(A)=P\left\{\left(X_{n+1}, \bar{X}_{n+1}\right) \in A \mid\right.$ $\left.\left(X_{n}, \bar{X}_{n}\right)=(x, y)\right\}$ of the coupled chain $\left(X_{n}, \bar{X}_{n}\right)_{n \in \mathbb{N}_{0}}$ taking values in $X^{2}$ can be decomposed (see [11]) in the following way:

$$
\mathbf{B}_{x, y}=\mathbf{Q}_{x, y}+\mathbf{R}_{x, y},
$$

where the subprobability measures $\mathbf{Q}_{x, y}$ are contractive, i.e. for some constant $\alpha \in(0,1) \int_{X^{2}} d(u, v) \mathbf{Q}_{x, y}(d u, d v) \leqslant \alpha d(x, y)$.

Since the transition probabilities for (1.1) can be mutually singular even for very close points, one cannot expect that the chains $\left(X_{n}\right)_{n \in \mathbb{N}_{0}}$ and $\left(\bar{X}_{n}\right)_{n \in \mathbb{N}_{0}}$ couple in finite time (i.e. $X_{n}=\bar{X}_{n}$ for some $n \in \mathbb{N}_{0}$ ) as in classical coupling constructions $([21])$ leading to convergence in the total variation norm. On the contrary, they only couple at infinity (i.e. $d\left(X_{n}, \bar{X}_{n}\right) \rightarrow 0$ as $\left.n \rightarrow \infty\right)$ so this method is sometimes called asymptotic coupling $([13])$ and gives convergence in the weak topology.

There are two reasons which make random iterations with place dependent probabilities in some sense the critical case. First, when passing from constant to place dependent probabilities one loses the backward iterations argument, which combined with mutual singularity of transition probabilities makes establishing exponential ergodicity a difficult task. Secondly, such iterations are a simplest example of systems where exponential ergodicity must be understood in the sense of convergence in the weak topology. Another example is much more sophisticated: stochastic partial differential equations (SPDEs). In both cases the main difficulty is the same: mutual singularity of transition probabilities. The emerging theory of weak exponential ergodicity brings many results which can be found in the vast SPDE literature (see [19], [20], [12], [34]), some of them ([13]) indicating analogies to the existing theory of strong ergodicity summarized in [23]. However, to our best knowledge, none of these results applies in our case.

One of the most important consequences of exponential ergodicity (understood here in the sense of the weak topology) is that it implies the Law of Large Num- 
bers and the Central Limit Theorem. Shirikyan [27] showed that if the process is exponentially ergodic in a norm equivalent to the Fortet-Mourier norm then the strong version of LLN holds. Since the convergence we obtain in this paper is in the Fortet-Mourier norm, Shirikyan's result can be applied in our case. Establishing the CLT is a much more delicate problem. As proved by Komorowski and Walczuk [18], exponential ergodicity implies the CLT, but they use the stronger Wasserstein norm. Recent results [14], [16] establish the CLT and exponential ergodicity simultaneously, using methods similar to ours. It is thus reasonable to expect that a general theorem in the spirit of Komorowski and Walczuk and involving the Fortet-Mourier norm can be formulated.

The paper is organized as follows. In Section 2 we formulate and prove a theorem which yields exponential convergence to an invariant measure for a class of Markov chains. This theorem is applied in Section 3 to chains generated by random iteration of functions. In Section 4 we discuss a special class of such functions, random affine transformations on $\mathbb{R}^{d}$, thus generalizing the notion of perpetuity to the place dependent case.

\section{AN EXPONENTIAL CONVERGENCE RESULT}

2.1. Notation and basic definitions. Let $(X, d)$ be a Polish space, i.e. a complete and separable metric space, and denote by $\mathcal{B}_{X}$ the $\sigma$-algebra of Borel subsets of $X$. We denote by $B_{b}(X)$ the space of bounded Borel-measurable functions equipped with the supremum norm, and $C_{b}(X)$ stands for the subspace of bounded continuous functions. Let $\mathcal{M}_{\mathrm{fin}}(X)$ and $\mathcal{M}_{1}(X)$ be the sets of Borel measures on $X$ such that $\mu(X)<\infty$ for $\mu \in \mathcal{M}_{\text {fin }}(X)$ and $\mu(X)=1$ for $\mu \in \mathcal{M}_{1}(X)$. The elements $\mu \in \mathcal{M}_{1}(X)$ are called probability measures, and the elements $\mu \in \mathcal{M}_{\text {fin }}(X)$ for which $\mu(X) \leqslant 1$ are called subprobability measures. We denote by supp $\mu$ the support of the measure $\mu$. We also define

$$
\mathcal{M}_{L}(X)=\left\{\mu \in \mathcal{M}_{1}(X): \int_{X} L(x) \mu(d x)<\infty\right\}
$$

where $L: X \rightarrow[0, \infty)$ is an arbitrary Borel measurable function, and

$$
\mathcal{M}_{1}^{1}(X)=\left\{\mu \in \mathcal{M}_{1}(X): \int_{X} d(\bar{x}, x) \mu(d x)<\infty\right\}
$$

where $\bar{x} \in X$ is fixed. By the triangle inequality the definition of $\mathcal{M}_{1}^{1}(X)$ is independent of the choice of $\bar{x}$.

The space $\mathcal{M}_{1}(X)$ is equipped with the Fortet-Mourier metric:

$$
\left\|\mu_{1}-\mu_{2}\right\|_{\mathrm{FM}}=\sup \left\{\left|\int_{X} f(x)\left(\mu_{1}-\mu_{2}\right)(d x)\right|: f \in \mathcal{F}\right\},
$$


where $\mathcal{F}=\left\{f \in C_{b}(X):|f(x)-f(y)| \leqslant 1\right.$ and $|f(x)| \leqslant 1$ for $\left.x, y \in X\right\}$. The space $\mathcal{M}_{1}^{1}(X)$ is equipped with the Wasserstein metric:

$$
\left\|\mu_{1}-\mu_{2}\right\|_{\mathrm{W}}=\sup \left\{\left|\int_{X} f(x)\left(\mu_{1}-\mu_{2}\right)(d x)\right|: f \in \mathcal{W}\right\}
$$

where $\mathcal{W}=\left\{f \in C_{b}(X):|f(x)-f(y)| \leqslant 1\right.$ for $\left.x, y \in X\right\}$. Let $\|\cdot\|$ denote the total variation norm. If a measure $\mu$ is nonnegative then $\|\mu\|$ is simply the total mass of $\mu$. In Section 4 we will use Euclidean norm $|\cdot|$ in $\mathbb{R}^{d}$ and the operator norm $\|\cdot\|_{\text {op }}$ given by $\|m\|_{\text {op }}=\sup \left\{|m x|: x \in \mathbb{R}^{d},|x|=1\right\}$.

Let $P: B_{b}(X) \rightarrow B_{b}(X)$ be a Markov operator, i.e. a linear operator satisfying $P \mathbf{1}_{X}=\mathbf{1}_{X}$ and $P f \geqslant 0$ if $f \geqslant 0$. Denote by $P^{*}$ the dual operator, i.e. the operator $P^{*}: \mathcal{M}_{\text {fin }}(X) \rightarrow \mathcal{M}_{\text {fin }}(X)$ defined as follows:

$$
P^{*} \mu(A):=\int_{X} P \mathbf{1}_{A}(x) \mu(d x) \quad \text { for } A \in \mathcal{B}_{X}
$$

We say that a measure $\mu_{*} \in \mathcal{M}_{1}(X)$ is invariant for $P$ if $\int_{X} P f(x) \mu_{*}(d x)=$ $\int_{X} f(x) \mu_{*}(d x)$ for every $f \in B_{b}(X)$, or equivalently $P^{*} \mu_{*}=\mu_{*}$. We denote by $\left\{\mathbf{P}_{x}: x \in X\right\}$ a transition probability function for $P$, i.e. a family of measures $\mathbf{P}_{x} \in \mathcal{M}_{1}(X)$ for $x \in X$ such that the map $x \mapsto \mathbf{P}_{x}(A)$ is measurable for every $A \in \mathcal{B}_{X}$ and $P f(x)=\int_{X} f(y) \mathbf{P}_{x}(d y)$ for $x \in X$ and $f \in B_{b}(X)$, or equivalently $P^{*} \mu(A)=\int_{X} \mathbf{P}_{x}(A) \mu(d x)$ for $A \in \mathcal{B}_{X}$ and $\mu \in \mathcal{M}_{\text {fin }}(X)$.

\subsection{Formulation of the theorem}

Definition 2.1. A coupling for $\left\{\mathbf{P}_{x}: x \in X\right\}$ is a family $\left\{\mathbf{B}_{x, y}: x, y \in X\right\}$ of probability measures on $X \times X$ such that for every $B \in \mathcal{B}_{X^{2}}$ the map $X^{2} \ni$ $(x, y) \mapsto \mathbf{B}_{x, y}(B)$ is measurable and

$$
\mathbf{B}_{x, y}(A \times X)=\mathbf{P}_{x}(A), \quad \mathbf{B}_{x, y}(X \times A)=\mathbf{P}_{y}(A)
$$

for all $x, y \in X$ and $A \in \mathcal{B}_{X}$.

In the following we assume (see [11]) that there exists a family $\left\{\mathbf{Q}_{x, y}\right.$ : $x, y \in X\}$ of subprobability measures on $X^{2}$ such that the map $(x, y) \mapsto \mathbf{Q}_{x, y}(B)$ is measurable for all $B \in \mathcal{B}_{X^{2}}$ and $\mathbf{Q}_{x, y}(A \times X) \leqslant \mathbf{P}_{x}(A)$ and $\mathbf{Q}_{x, y}(X \times A) \leqslant$ $\mathbf{P}_{y}(A)$ for all $x, y \in X$ and $A \in \mathcal{B}_{X}$.

The measures $\left\{\mathbf{Q}_{x, y}: x, y \in X\right\}$ allow us to construct a coupling for $\left\{\mathbf{P}_{x}: x \in\right.$ $X\}$. Define on $X^{2}$ a family $\left\{\mathbf{R}_{x, y}: x, y \in X\right\}$ of measures which on rectangles $A \times B$ are given by

$$
\mathbf{R}_{x, y}(A \times B)=\frac{1}{1-\mathbf{Q}_{x, y}\left(X^{2}\right)}\left(\mathbf{P}_{x}(A)-\mathbf{Q}_{x, y}(A \times X)\right)\left(\mathbf{P}_{y}(B)-\mathbf{Q}_{x, y}(X \times B)\right)
$$


if $\mathbf{Q}_{x, y}\left(X^{2}\right)<1$ and $\mathbf{R}_{x, y}(A \times B)=0$ otherwise. A simple computation shows that the family $\left\{\mathbf{B}_{x, y}: x, y \in X\right\}$ of measures on $X^{2}$ defined by

$$
\mathbf{B}_{x, y}=\mathbf{Q}_{x, y}+\mathbf{R}_{x, y} \quad \text { for } x, y \in X
$$

is a coupling for $\left\{\mathbf{P}_{x}: x \in X\right\}$.

Now we list our assumptions on the Markov operator $P$ and the transition subprobabilities $\left\{\mathbf{Q}_{x, y}: x, y \in X\right\}$.

A0. $P$ is a Feller operator, i.e. $P\left(C_{b}(X)\right) \subset C_{b}(X)$.

A1. There exists a Lyapunov function for $P$, i.e. a continuous function $L: X \rightarrow$ $[0, \infty)$ such that $L$ is bounded on bounded sets, $\lim _{x \rightarrow \infty} L(x)=+\infty$ (for bounded $X$ this condition is omitted) and for some $\lambda \in(0,1)$ and $c>0$

$$
P L(x) \leqslant \lambda L(x)+c \quad \text { for } x \in X .
$$

A2. There exist $F \subset X^{2}$ and $\alpha \in(0,1)$ such that $\operatorname{supp} \mathbf{Q}_{x, y} \subset F$ and

$$
\int_{X^{2}} d(u, v) \mathbf{Q}_{x, y}(d u, d v) \leqslant \alpha d(x, y) \quad \text { for }(x, y) \in F .
$$

A3. There exist $\delta, l>0$ and $\nu \in(0,1]$ such that

$$
\begin{aligned}
& 1-\left\|\mathbf{Q}_{x, y}\right\| \leqslant l d(x, y)^{\nu} \quad \text { and } \\
& \mathbf{Q}_{x, y}\left(\left\{(u, v) \in X^{2}: d(u, v)<\alpha d(x, y)\right\}\right) \geqslant \delta
\end{aligned}
$$

for $(x, y) \in F$.

A4. There exist $\beta \in(0,1)$ and $\tilde{C}, R>0$ such that for

$$
\kappa\left(\left(x_{n}, y_{n}\right)_{n \in \mathbb{N}_{0}}\right)=\inf \left\{n \in \mathbb{N}_{0}:\left(x_{n}, y_{n}\right) \in F \text { and } L\left(x_{n}\right)+L\left(y_{n}\right)<R\right\},
$$

where $\left(x_{n}, y_{n}\right)_{n \in \mathbb{N}_{0}}$ is a sequence of elements of $X \times X$, we have

$$
\mathbb{E}_{x, y} \beta^{-\kappa} \leqslant \tilde{C} \quad \text { whenever } L(x)+L(y)<\frac{4 c}{1-\lambda},
$$

where $\mathbb{E}_{x, y}$ denotes the expectation with respect to the chain starting from $(x, y)$ and with transition function $\left\{\mathbf{B}_{x, y}: x, y \in X\right\}$.

Before proceeding to the formulation of the theorem we briefly comment on the above conditions. Assumption A0 is standard. Existence of a Lyapunov function in A1 allows one to reduce the dynamics to some bounded region of $X$. Assumption A2 is the well known contraction on average condition, written in the language of coupling. The measures $\mathbf{Q}_{x, y}$ are thus the contractive part of coupled transition probabilities $\mathbf{B}_{x, y}$ with $\mathbf{R}_{x, y}$ being the uncontrollable part. Some such condition seems to be necessary for exponential ergodicity (see [25]). The first part 
of $\mathbf{A} \mathbf{3}$ means that the total mass of the contractive part $\mathbf{Q}_{x, y}$ is close to 1 for $x$ close to $y$. Some higher order regularity is necessary here to ensure the uniqueness of the invariant measure (see [30]). The second part of A3 guarantees that some part of the contractive-on-average measure $\mathbf{Q}_{x, y}$ is contractive in the strict sense. Condition A4 means that the dynamics quickly enters the domain $F$ of contractivity. In this paper we discuss Markov chains generated by random iteration of functions for which always $F=X^{2}$ and $L(x)=d(x, \bar{x})$ with some fixed $\bar{x} \in X$, so A4 is trivially fulfilled when $R=\frac{4 c}{1-\lambda}$. There are, however, examples of random dynamical systems for which $F$ is a proper subset of $X^{2}$. Indeed, in contractive Markov systems introduced by I. Werner [33] we have $X=\sum_{i=1}^{n} X_{i}$ but $F=\sum_{i=1}^{n} X_{i} \times X_{i}$. They are studied in [29].

Now we formulate the main result of this section. Its proof is given in Section 2.4.

Theorem 2.1. Assume A0-A4. Then the operator $P$ has a unique invariant measure $\mu_{*} \in \mathcal{M}_{L}(X)$, which is attractive, i.e.

$$
\lim _{n \rightarrow \infty} \int_{X} P^{n} f(x) \mu(d x)=\int_{X} f(x) \mu(d x) \quad \text { for } f \in C_{b}(X), \mu \in \mathcal{M}_{1}(X) .
$$

Moreover, there exist $q \in(0,1)$ and $C>0$ such that

$$
\left\|P^{* n} \mu-\mu_{*}\right\|_{\mathrm{FM}} \leqslant q^{n} C\left(1+\int_{X} L(x) \mu(d x)\right)
$$

for $\mu \in \mathcal{M}_{L}(X)$ and $n \in \mathbb{N}$.

REMARK. In [13, Theorem 4.8], sufficient conditions are given for the existence of a unique exponentially attractive invariant measure for a continuous-time Markov semigroup $\{P(t)\}_{t \geqslant 0}$, which do not refer to coupling. One of the assumptions is that there exists a distance-like (i.e. symmetric, lower semicontinuous and vanishing only on the diagonal) function $d: X \times X \rightarrow[0,1]$ which is contractive for some $P\left(t_{*}\right)$, i.e. there exists $\alpha<1$ such that for all $x, y \in X$ with $d(x, y)<1$ we have $d(\mathcal{P}(x, \cdot), \mathcal{P}(y, \cdot)) \leqslant \alpha d(x, y)$, where $\mathcal{P}(\cdot, \cdot): X \times \mathcal{B}_{X} \rightarrow[0,1]$ is a transition kernel for $P\left(t_{*}\right)$. This assumption is stronger than $\mathbf{A 2}$, since the measures $\mathbf{R}_{x, y}$ in 2.1 need not be contractive (i.e. need not satisfy $\int_{X^{2}} d(u, v) \mathbf{R}_{x, y}(d u, d v)$ $\leqslant \alpha d(u, v)$ ) for any distance-like function $d$.

2.3. Measures on the pathspace. For fixed $\left(x_{0}, y_{0}\right) \in X^{2}$ the next step of a chain with transition probability function $\mathbf{B}_{x, y}=\mathbf{Q}_{x, y}+\mathbf{R}_{x, y}$ can be drawn according to $\mathbf{Q}_{x_{0}, y_{0}}$ or according to $\mathbf{R}_{x_{0}, y_{0}}$. To distinguish these two cases we introduce (see [11]) the augmented space $\widehat{X}=X^{2} \times\{0,1\}$ and the transition function $\left\{\widehat{\mathbf{B}}_{x, y, \theta}:(x, y, \theta) \in \widehat{X}\right\}$ on $\widehat{X}$ given by $\widehat{\mathbf{B}}_{x, y, \theta}=\widehat{\mathbf{Q}}_{x, y, \theta}+\widehat{\mathbf{R}}_{x, y, \theta}$, where $\widehat{\mathbf{Q}}_{x, y, \theta}=\mathbf{Q}_{x, y} \times \delta_{1}$ and $\widehat{\mathbf{R}}_{x, y, \theta}=\mathbf{R}_{x, y} \times \delta_{0}$. The parameter $\theta \in\{0,1\}$ is responsible for choosing $\mathbf{Q}_{x, y}$ or $\mathbf{R}_{x, y}$. If a Markov chain with transition function 
$\left\{\widehat{\mathbf{B}}_{x, y, \theta}:(x, y, \theta) \in \widehat{X}\right\}$ stays in the set $X^{2} \times\{1\}$ at time $n$ it means that the last step was drawn according to $\mathbf{Q}_{u, v}$ for some $(u, v) \in X^{2}$.

For every $x \in X$ finite-dimensional distributions $\mathbf{P}_{x}^{0, \ldots, n} \in \mathcal{M}_{1}\left(X^{n+1}\right)$ are defined by

$$
\mathbf{P}_{x}^{0, \ldots, n}(B)=\int_{X} \delta_{x}\left(d x_{0}\right) \int_{X} \mathbf{P}_{x_{1}}\left(d x_{2}\right) \ldots \int_{X} \mathbf{P}_{x_{n-1}}\left(d x_{n}\right) \mathbf{1}_{B}\left(x_{0}, \ldots, x_{n}\right)
$$

for $n \in \mathbb{N}_{0}$ and $B \in \mathcal{B}_{X^{n+1}}$, where $\delta_{x}$ is the Dirac measure at $x$. By the Kolmogorov extension theorem we obtain a measure $\mathbf{P}_{x}^{\infty}$ on the pathspace $X^{\infty}$. Similarly we define measures $\mathbf{B}_{x, y}^{\infty}, \widehat{\mathbf{B}}_{x, y, \theta}^{\infty}$ on $(X \times X)^{\infty}$ and $\widehat{X}^{\infty}$. These measures have the following interpretation. Consider the Markov chain $\left(X_{n}, Y_{n}\right)_{n \in \mathbb{N}_{0}}$ on $X \times X$, starting from $\left(x_{0}, y_{0}\right)$, with transition function $\left\{\mathbf{B}_{x, y}: x, y \in X\right\}$, obtained by the canonical Kolmogorov construction, i.e. $\Omega=(X \times X)^{\infty}$ is the sample space equipped with the probability measure $\mathbb{P}=\mathbf{B}_{x_{0}, y_{0}}^{\infty}, X_{n}(\omega)=x_{n}, Y_{n}(\omega)=y_{n}$, where $\omega=\left(x_{k}, y_{k}\right)_{k \in \mathbb{N}_{0}} \in \Omega$ and $n \in \mathbb{N}_{0}$. Then $\left(X_{n}\right)_{n \in \mathbb{N}_{0}},\left(Y_{n}\right)_{n \in \mathbb{N}_{0}}$ are Markov chains in $X$, starting from $x_{0}$ and $y_{0}$, with transition function $\left\{\mathbf{P}_{x}: x \in X\right\}$, and $\mathbf{P}_{x}^{\infty}, \mathbf{P}_{y}^{\infty}$ are their measures on the pathspace $X^{\infty}$.

In this paper we often consider marginals of measures on the pathspace. If $\mu$ is a measure on a measurable space $X$ and $f: X \rightarrow Y$ is a measurable map, then $f^{\#} \mu$ is the measure on $Y$ defined by $f^{\#} \mu(A)=\mu\left(f^{-1}(A)\right)$. So, if we denote by $p r$ the projection map from a product space to its component, then $p r^{\#} \mu$ is simply the marginal of $\mu$ on that component.

In the following we consider Markov chains on $\widehat{X}$ with transition function $\left\{\widehat{\mathbf{B}}_{x, y, \theta}: x, y \in X, \theta \in\{0,1\}\right\}$. We adopt the convention that $\theta_{0}=1$, so the chain always starts from $X^{2} \times\{1\}$, and define $\widehat{\mathbf{B}}_{x, y}^{\infty}:=\widehat{\mathbf{B}}_{x, y, 1}^{\infty}$.

For $b \in \mathcal{M}_{\text {fin }}\left(X^{2}\right)$ we write $\widehat{\mathbf{B}}_{b}^{\infty}(B)=\int_{X} \widehat{\mathbf{B}}_{x, y}^{\infty}(B) b(d x, d y)$ for $B \in \mathcal{B}_{\widehat{X}^{\infty}}$, $\mathbf{Q}_{b}(A)=\int_{X^{2}} \mathbf{Q}_{x, y}(A) b(d x, d y)$ for $A \in \mathcal{B}_{X^{2}}$ and $\mathbf{Q}_{x, y}^{n}(A)=\mathbf{Q}_{\mathbf{Q}_{x, y}^{n-1}}(A)$ for $A \in \mathcal{B}_{X^{2}}$. When studying the asymptotics of a chain $\left(X_{n}\right)_{n \in \mathbb{N}_{0}}$ with transition function $\left\{\mathbf{P}_{x}: x \in X\right\}$ it is particularly interesting whether a coupled chain $\left(X_{n}, Y_{n}\right)_{n \in \mathbb{N}_{0}}$ is moving only according to the contractive part $\mathbf{Q}_{x, y}$ of the transition function $\mathbf{B}_{x, y}$. For every subprobability measure $b \in \mathcal{M}_{\text {fin }}\left(X^{2}\right)$ we define finite-dimensional subprobability distributions $\mathbf{Q}_{b}^{0, \ldots, n} \in \mathcal{M}_{\text {fin }}\left((X \times X)^{n+1}\right)$ by

$$
\begin{aligned}
\mathbf{Q}_{b}^{0, \ldots, n}(B)=\int_{X^{2}} b\left(d x_{0}, d y_{0}\right) \int_{X^{2}} \mathbf{Q}_{x_{0}, y_{0}}\left(d x_{1}, d y_{1}\right) \ldots \\
\quad \cdots \int_{X^{2}} \mathbf{Q}_{x_{n-1}, y_{n-1}}\left(d x_{n}, d y_{n}\right) \mathbf{1}_{B}\left(\left(x_{0}, y_{0}\right), \ldots,\left(x_{n}, y_{n}\right)\right),
\end{aligned}
$$

where $B \in \mathcal{B}_{(X \times X)^{n+1}}$ and $n \in \mathbb{N}_{0}$. For $n \geqslant 1$ define $\iota_{n}:(X \times X)^{n} \rightarrow \widehat{X}^{n}$ by

$$
\iota_{n}\left(\left(x_{1}, y_{1}\right), \ldots,\left(x_{n}, y_{n}\right)\right)=\left(\left(x_{1}, y_{1}, 1\right), \ldots,\left(x_{n}, y_{n}, 1\right)\right)
$$


and $\widehat{\mathbf{Q}}_{b}^{0, \ldots, n}=\iota_{n}^{\#} \mathbf{Q}_{b}^{0, \ldots, n}$. Since $\mathbf{Q}_{b}^{0, \ldots, n}\left((X \times X)^{n+1}\right)>\mathbf{Q}_{b}^{0, \ldots, n+1}\left((X \times X)^{n+2}\right)$, the family $\left\{\widehat{\mathbf{Q}}_{b}^{0, \ldots, n}: n \in \mathbb{N}_{0}\right\}$ is not consistent and we cannot use the Kolmogorov extension theorem to obtain a measure on the whole pathspace $\widehat{X}^{\infty}$. However, defining for every $b \in \mathcal{M}_{\text {fin }}\left(X^{2}\right)$ a measure $\mathbf{Q}_{b}^{\infty} \in \mathcal{M}_{\text {fin }}\left(\widehat{X}^{\infty}\right)$ by $\mathbf{Q}_{b}^{\infty}(B)=\widehat{\mathbf{B}}_{b}^{\infty}\left(B \cap\left(X^{2} \times\{1\}\right)^{\infty}\right)$ for $B \in \mathcal{B}_{\widehat{X}^{\infty}}$, one can easily check that for every cylindrical set $B=A \times \widehat{X}^{\infty}$ with $A \in \mathcal{B}_{\widehat{X}^{n+1}}$, we have

$$
\mathbf{Q}_{b}^{\infty}(B)=\lim _{n \rightarrow \infty} \widehat{\mathbf{Q}}_{b}^{0, \ldots, n}(A) .
$$

2.4. Proof of Theorem 2.1. Before proceeding to the proof of Theorem 2.1 we formulate two lemmas. The first one is partially inspired by the reasoning found in [26].

LEMMA 2.1. Let $Y$ be a metric space and let $\left(Y_{n}^{y}\right)_{n \in \mathbb{N}_{0}}$ be a family of Markov chains indexed by the starting point $y \in Y$, with common transition function $\left\{\pi_{y}\right.$ : $y \in Y\}$. Let $V: Y \rightarrow[0, \infty)$ be a Lyapunov function for $\left\{\pi_{y}: y \in Y\right\}$. Assume that for some bounded and measurable $A \subset Y$ there exist $\lambda \in(0,1)$ and $C_{\rho}>0$ such that for

$$
\rho\left(\left(y_{n}\right)_{n \in \mathbb{N}_{0}}\right)=\inf \left\{n \geqslant 1: y_{n} \in A\right\}
$$

we have

$$
\mathbb{E}_{y} \lambda^{-\rho} \leqslant C_{\rho}\left(V\left(y_{0}\right)+1\right) \quad \text { for } y \in Y,
$$

where $\mathbb{E}_{y}$ is the expectation with respect to the measure $\mathbb{P}_{y}$ on $Y^{\infty}$ induced by $\left(Y_{n}^{y}\right)_{n \in \mathbb{N}_{0}}$. Moreover, assume that for some measurable $B \subset Y$ and

$$
\epsilon\left(\left(y_{n}\right)_{n \in \mathbb{N}_{0}}\right)=\inf \left\{n \geqslant 1: y_{n} \notin B\right\}
$$

there exist constants $p, \beta \in(0,1)$ and $C_{\epsilon}>0$ such that

$$
\mathbb{P}_{y}\left(\left\{\left(y_{n}\right)_{n \in \mathbb{N}_{0}}: \forall_{n \geqslant 1} y_{n} \in B\right\}\right)>p \quad \text { and } \quad \mathbb{E}_{y} \mathbf{1}_{\{\epsilon<\infty\}} \beta^{-\epsilon} \leqslant C_{\epsilon}
$$

for every $y \in A$. Then there exist $\gamma \in(0,1)$ and $C>0$ such that for

$$
\tau\left(\left(y_{n}\right)_{n \in \mathbb{N}_{0}}\right)=\inf \left\{n \geqslant 1: \forall_{k \geqslant n} y_{k} \in B\right\}
$$

we have

$$
\mathbb{E}_{y} \gamma^{-\tau} \leqslant C(V(y)+1) \quad \text { for } y \in Y .
$$

Proof. Define $\kappa=\epsilon+\rho \circ T_{\epsilon}$, where $T_{n}\left(\left(y_{k}\right)_{k \in \mathbb{N}_{0}}\right)=\left(y_{k+n}\right)_{k \in \mathbb{N}_{0}}$. Fix $y \in Y$, $\alpha \in(0,1)$ and $r>1$ such that $(\lambda \alpha)^{-1 /(r-1)}<\beta^{-1}$. The strong Markov property and the Hölder inequality for every $y \in Y$ give

$$
\begin{aligned}
\mathbb{E}_{y} \mathbf{1}_{\{\epsilon<\infty\}} \lambda^{-\frac{\kappa}{r}} & \leqslant\left[\mathbb{E}_{y}\left(\mathbf{1}_{\{\epsilon<\infty\}}(\lambda \alpha)^{-\frac{\epsilon}{r}}\right)^{\frac{r}{r-1}}\right]^{\frac{r-1}{r}}\left[\mathbb{E}_{y}\left(\mathbf{1}_{\{\epsilon<\infty\}} \alpha^{\epsilon} \lambda^{-\rho \circ T_{\epsilon}}\right)\right]^{\frac{1}{r}} \\
& \leqslant\left(\mathbb{E}_{y} \mathbf{1}_{\{\epsilon<\infty\}} \beta^{-\epsilon}\right)^{\frac{r-1}{r}}\left[\mathbb{E}_{y}\left(\mathbf{1}_{\{\epsilon<\infty\}} \alpha^{\epsilon} \mathbb{E}_{y}\left(\lambda^{-\rho \circ T_{\epsilon}} \mid \mathcal{F}_{\epsilon}\right)\right)\right]^{\frac{1}{r}} \\
& =\left(\mathbb{E}_{y} \mathbf{1}_{\{\epsilon<\infty\}} \beta^{-\epsilon}\right)^{\frac{r-1}{r}}\left[\mathbb{E}_{y}\left(\mathbf{1}_{\{\epsilon<\infty\}} \alpha^{\epsilon} \mathbb{E}_{Y_{\epsilon}^{y}}\left(\lambda^{-\rho}\right)\right)\right]^{\frac{1}{r}} \\
& \leqslant\left(\mathbb{E}_{y} \mathbf{1}_{\{\epsilon<\infty\}} \beta^{-\epsilon}\right)^{\frac{r-1}{r}}\left[\mathbb{E}_{y}\left(\mathbf{1}_{\{\epsilon<\infty\}} \alpha^{\epsilon} C_{\rho}\left(V\left(Y_{\epsilon}^{y}\right)+1\right)\right)\right]^{\frac{1}{r}},
\end{aligned}
$$


where $\mathcal{F}_{\epsilon}$ is the $\sigma$-algebra generated by $\epsilon$. Since $\sup _{y \in A} V(y)<\infty$ and $V$ satisfies

$$
\mathbb{E}_{y}\left(\mathbf{1}_{\{\epsilon<\infty\}} \alpha^{\epsilon} V\left(Y_{\epsilon}^{y}\right)\right) \leqslant C_{1}(V(y)+1) \quad \text { for } y \in Y,
$$

for some $C_{1}>0$, taking $c=\lambda^{1 / r}$ we obtain $\mathbb{E}_{y} \mathbf{1}_{\{\epsilon<\infty\}} c^{-\kappa} \leqslant C_{2}$ whenever $y \in A$, for some constant $C_{2}>0$. Define $\epsilon_{0}=0, \kappa_{0}=\rho$ and

$$
\begin{aligned}
& \epsilon_{n}=\kappa_{n-1}+\epsilon \circ T_{\kappa_{n-1}}, \\
& \kappa_{n}=\kappa_{n-1}+\kappa \circ T_{\kappa_{n-1}} \quad \text { for } n \geqslant 1 .
\end{aligned}
$$

Observe that $Y_{\kappa_{n}}^{y} \in A, Y_{\epsilon_{n}}^{y} \notin B, \epsilon_{n} \leqslant \kappa_{n} \leqslant \epsilon_{n+1}$ and $\kappa_{n} \nearrow \infty$. We have

$$
\begin{aligned}
\mathbb{E}_{y} \mathbf{1}_{\left\{\kappa_{n+1}<\infty\right\}} c^{-\kappa_{n+1}} & =\mathbb{E}_{y}\left[\mathbf{1}_{\left\{\kappa_{n}<\infty\right\}} c^{-\kappa_{n}} \mathbb{E}_{y}\left(\left(\mathbf{1}_{\{\kappa<\infty\}} c^{-\kappa}\right) \circ T_{\kappa_{n}} \mid \mathcal{F}_{\kappa_{n}}\right)\right] \\
& =\mathbb{E}_{y}\left[\mathbf{1}_{\left\{\kappa_{n}<\infty\right\}} c^{-\kappa_{n}} \mathbb{E}_{Y_{\kappa_{n}}^{y}}\left(\mathbf{1}_{\{\kappa<\infty\}} c^{-\kappa}\right)\right] \\
& \leqslant C_{2} \mathbb{E}_{y} \mathbf{1}_{\left\{\kappa_{n}<\infty\right\}} c^{-\kappa_{n}}
\end{aligned}
$$

and thus

$$
\mathbb{E}_{y} \mathbf{1}_{\left\{\kappa_{n}<\infty\right\}} c^{-\kappa_{n}} \leqslant C_{2}^{n} C_{\rho}(V(y)+1) \quad \text { for } y \in Y .
$$

Define $E=Y^{\infty} \backslash\left(Y \times B^{\infty}\right)$ and $B_{n}=\left\{\epsilon_{n}<\infty\right\}$. Observe that $B_{n+1}=T_{\kappa_{n}}^{-1} E$ and $B_{n} \in \mathcal{F}_{\epsilon_{n}} \subset \mathcal{F}_{\kappa_{n}}$. For $y \in Y$ we have

$$
\begin{aligned}
\mathbb{P}_{y}\left(B_{n+1}\right) & =\mathbb{P}_{y}\left(B_{n} \cap B_{n+1}\right)=\mathbb{E}_{y}\left(\mathbf{1}_{B_{n}} \mathbb{E}_{y}\left(\mathbf{1}_{E} \circ T_{\kappa_{n}} \mid \mathcal{F}_{\kappa_{n}}\right)\right) \\
& =\int_{B_{n}} \mathbb{P}_{x_{\kappa_{n}}}(E) \mathbb{P}_{y}(d x) \leqslant(1-p) \mathbb{P}_{y}\left(B_{n}\right),
\end{aligned}
$$

where $x=\left(x_{0}, x_{1}, \ldots\right) \in Y^{\infty}$. It follows that

$$
\mathbb{P}_{y}\left(B_{n}\right) \leqslant(1-p)^{n} \quad \text { for } y \in Y, n \geqslant 1 .
$$

Define $\widehat{\tau}\left(\left(y_{n}\right)_{n \in \mathbb{N}_{0}}\right)=\inf \left\{n \geqslant 1: y_{n} \in A, \forall_{k>n} y_{k} \in B\right\}, D_{0}=\left\{\widehat{\tau}=\kappa_{0}\right\}$ and $D_{n}=\left\{\kappa_{n-1}<\widehat{\tau} \leqslant \kappa_{n}<\infty\right\}$, for $n \geqslant 1$. Since $B_{n}=\left\{\widehat{\tau}>\kappa_{n-1}\right\}$, we have $D_{n} \subset B_{n}$ for $n \geqslant 0$ and $\mathbb{P}_{y}(\widehat{\tau}=\infty)=\lim _{n \rightarrow \infty} \mathbb{P}_{y}\left(\widehat{\tau}>\kappa_{n-1}\right)=$ $\lim _{n \rightarrow \infty} \mathbb{P}_{y}\left(B_{n}\right) \leqslant \lim _{n \rightarrow \infty}(1-p)^{n}=0$ for $y \in Y$. Finally, by the Hölder inequality, for $s>1$ and $y \in Y$ we obtain

$$
\begin{aligned}
\mathbb{E}_{y} \lambda^{-\widehat{\tau} / s} & \leqslant \sum_{n=0}^{\infty} \mathbb{E}_{y}\left(\mathbf{1}_{\left\{\kappa_{n}<\infty\right\}} \lambda^{-\kappa_{n} / s} \mathbf{1}_{D_{n}}\right) \\
& \leqslant \sum_{n=0}^{\infty}\left[\mathbb{E}_{y} \mathbf{1}_{\left\{\kappa_{n}<\infty\right\}} \lambda^{-\kappa_{n}}\right]^{1 / s} \mathbb{P}_{y}\left(D_{n}\right)^{1-1 / s} \\
& \leqslant \sum_{n=0}^{\infty}\left[C_{2}^{n} C_{\rho}(V(y)+1)\right]^{1 / s}(1-p)^{n(1-1 / s)} \\
& \leqslant C_{\rho}^{1 / s}(1+V(y)) \sum_{n=0}^{\infty}\left[\left(\frac{C_{2}}{1-p}\right)^{1 / s}(1-p)\right]^{n} .
\end{aligned}
$$

Choosing sufficiently large s and setting $\gamma=\lambda^{1 / s}$ we have $\mathbb{E}_{y} \gamma^{-\widehat{\tau}} \leqslant C(1+V(y))$ for $y \in Y$. Since $\tau<\widehat{\tau}$, the proof is complete. 
LEMMA 2.2. Let $\left(Y_{n}^{y}\right)_{n \in \mathbb{N}_{0}}$ with $y \in Y$ be a family of Markov chains on a metric space $Y$. Suppose that $V: Y \rightarrow[0, \infty)$ is a Lyapunov function for their transition function $\left\{\pi_{y}: y \in Y\right\}$, i.e. there exist $a \in(0,1)$ and $b>0$ such that

$$
\int_{Y} V(x) \pi_{y}(d x) \leqslant a V(y)+b \quad \text { for } y \in Y \text {. }
$$

Then there exist $\lambda \in(0,1)$ and $\tilde{C}>0$ such that for

$$
\rho\left(\left(y_{k}\right)_{k \in \mathbb{N}_{0}}\right)=\inf \left\{k \geqslant 1: V\left(y_{k}\right)<\frac{2 b}{1-a}\right\}
$$

we have

$$
\mathbb{E}_{y} \lambda^{-\rho} \leqslant \tilde{C}\left(V\left(y_{0}\right)+1\right) \quad \text { for } y \in Y .
$$

Proof. Suppose that the chains $\left(Y_{n}^{y}\right)_{n \in \mathbb{N}_{0}}$ for $y \in Y$ are defined on a common probability space $(\Omega, \mathcal{F}, \mathbb{P})$. Fix $\alpha \in\left(\frac{1+a}{2}, 1\right)$ and set $V_{0}=\frac{b}{\alpha-a}$. Define $\tilde{\rho}\left(\left(y_{k}\right)_{k \in \mathbb{N}_{0}}\right)=\inf \left\{k \geqslant 1: V\left(y_{k}\right) \leqslant V_{0}\right\}$. Fix $y \in Y$. Let $\mathcal{F}_{n} \subset \mathcal{F}, n \in \mathbb{N}_{0}$, be the filtration induced by $\left(Y_{n}^{y}\right)_{n \in \mathbb{N}_{0}}$. Define

$$
A_{n}=\left\{\omega \in \Omega: V\left(Y_{i}^{y}(\omega)\right)>V_{0} \text { for } i=0,1, \ldots, n\right\}, \quad n \in \mathbb{N}_{0} .
$$

Observe that $A_{n+1} \subset A_{n}$ and $A_{n} \in \mathcal{F}_{n}$. By the definition of $V_{0}$ we have $\mathbf{1}_{A_{n}} \mathbb{E}\left(V\left(Y_{n+1}^{y}\right) \mid \mathcal{F}_{n}\right) \leqslant \mathbf{1}_{A_{n}}\left(a V\left(Y_{n}^{y}\right)+b\right)<\alpha \mathbf{1}_{A_{n}} V\left(Y_{n}^{y}\right) \mathbb{P}$-a.e. in $\Omega$. This gives

$$
\begin{aligned}
\int_{A_{n}} V\left(Y_{n}^{y}\right) d \mathbb{P} & \leqslant \int_{A_{n-1}} V\left(Y_{n}^{y}\right) d \mathbb{P}=\iint_{A_{n-1}} \mathbb{E}\left(V\left(Y_{n}^{y}\right) \mid \mathcal{F}_{n-1}\right) d \mathbb{P} \\
& \leqslant \int_{A_{n-1}}\left(a V\left(Y_{n-1}^{y}\right)+b\right) d \mathbb{P} \leqslant \alpha \int_{A_{n-1}} V\left(Y_{n-1}^{y}\right) d \mathbb{P} .
\end{aligned}
$$

By the Chebyshev inequality,

$$
\begin{aligned}
\mathbb{P}\left(V\left(Y_{0}^{y}\right)>V_{0}, \ldots, V\left(Y_{n}^{y}\right)>V_{0}\right)=\int_{A_{n-1}} \mathbb{P}\left(V\left(Y_{n}^{y}\right)>V_{0} \mid \mathcal{F}_{n-1}\right) d \mathbb{P} \\
\leqslant V_{0}^{-1} \int_{A_{n-1}} \mathbb{E}\left(V\left(Y_{n}^{y}\right) \mid \mathcal{F}_{n-1}\right) d \mathbb{P} \leqslant \alpha^{n-1} V_{0}^{-1}(a V(y)+b),
\end{aligned}
$$

thus for some $C>0$ we have

$$
\mathbb{P}_{y}(\tilde{\rho}>n) \leqslant \alpha^{n} C(V(y)+1), \quad n \in \mathbb{N}_{0} .
$$

Fix $\gamma \in(0,1)$ and observe that for $\lambda=\alpha^{\gamma}$ we have

$$
\begin{aligned}
\mathbb{E}_{y} \lambda^{-\tilde{\rho}} & \leqslant 2+\sum_{n=1}^{\infty} \mathbb{P}_{y}\left(\lambda^{-\tilde{\rho}}>n\right)=2+\sum_{n=1}^{\infty} \mathbb{P}_{y}\left(\tilde{\rho}>\log _{\alpha}\left(n^{-1 / \gamma}\right)\right) \\
& \leqslant 2+\sum_{n=1}^{\infty} \mathbb{P}_{y}\left(\tilde{\rho}>\left\lfloor\log _{\alpha}\left(n^{-1 / \gamma}\right)\right\rfloor\right) \leqslant 2+\frac{C(V(y)+1)}{\alpha} \sum_{n=1}^{\infty} n^{-1 / \gamma} \\
& =\tilde{C}(V(y)+1)
\end{aligned}
$$

for some $\tilde{C}$. Since $\rho \leqslant \tilde{\rho}$, the proof is finished. 
Proof of Theorem 2.1. For every $r>0$ define $D_{r}=\left\{(x, y) \in X^{2}\right.$ : $d(x, y)<r\}$.

STEP I. To simplify calculations define a new metric $\bar{d}(x, y)=d(x, y)^{\nu}$ and observe that for $\bar{D}_{r}=\left\{(x, y) \in X^{2}: \bar{d}(x, y)<r\right\}$ we have $D_{R}=\bar{D}_{\bar{R}}$ with $\bar{R}=R^{\nu}$. By the Jensen inequality, 2.2 takes the form

$$
\int_{X^{2}} \bar{d}(u, v) \mathbf{Q}_{x, y}(d u, d v) \leqslant \bar{\alpha} \bar{d}(x, y) \quad \text { for }(x, y) \in F,
$$

with $\bar{\alpha}=\alpha^{\nu}$. Assumption $\mathbf{A 3}$ implies that

$$
1-\left\|\mathbf{Q}_{x, y}\right\| \leqslant l \bar{d}(x, y) \quad \text { and } \quad \mathbf{Q}_{x, y}\left(D_{\bar{\alpha} \bar{d}(x, y)}\right) \geqslant \delta
$$

for $(x, y) \in F$.

STEP II. Observe that if $b \in \mathcal{M}_{\text {fin }}\left(X^{2}\right)$ satisfies $\operatorname{supp} b \subset F$ then 2.7 implies

$$
\left\|\mathbf{Q}_{b}\right\| \geqslant\|b\|-l \int_{X^{2}} \bar{d}(u, v) b(d u, d v) .
$$

Since $\operatorname{supp} b \subset F$, we have $\operatorname{supp} \mathbf{Q}_{b}^{0, \ldots, n} \subset F^{n+1}$. Iterating the above inequality and using 2.6, we obtain $\left\|\mathbf{Q}_{b}^{0, \ldots, n}\right\| \geqslant\|b\|-\frac{l}{1-\bar{\alpha}} \int_{X^{2}} \bar{d}(u, v) b(d u, d v)$. If $\operatorname{supp} b \subset\left\{(u, v) \in X^{2}: \bar{d}(u, v)<\frac{1-\bar{\alpha}}{2 l}\right\} \cap F$ then from 2.5$)$ it follows that

$$
\left\|\mathbf{Q}_{b}^{\infty}\right\| \geqslant \frac{1}{2}\|b\| .
$$

Set $R_{0}=\sup \{\bar{d}(x, y): L(x)+L(y)<R\}<\infty$ and $n_{0}=\min \left\{n \in \mathbb{N}_{0}:\right.$ $\left.\bar{\alpha}^{n} R_{0}<\frac{1-\bar{\alpha}}{2 l}\right\}$. Now 2.7 implies that for $(x, y) \in F$ such that $L(x)+L(y)<R$ we have

$$
\mathbf{Q}_{x, y}^{n_{0}}\left(\left\{(u, v) \in X^{2}: \bar{d}(u, v)<\frac{1-\bar{\alpha}}{2 l}\right\} \cap F\right)>\delta^{n_{0}}
$$

and finally $(2.8)$ gives

$$
\left\|\mathbf{Q}_{x, y}^{\infty}\right\| \geqslant \frac{1}{2} \delta^{n_{0}} .
$$

STEP III. Define $\tilde{\rho}\left(\left(x_{n}, y_{n}\right)_{n \in \mathbb{N}_{0}}\right)=\inf \left\{n \geqslant 1: L\left(x_{n}\right)+L\left(y_{n}\right)<\frac{4 c}{1-\lambda}\right\}$. Since $L(x)+L(y)$ is a Lyapunov function for a Markov chain in $X^{2}$ with transition probabilities $\left\{\mathbf{B}_{x, y}: x, y \in X\right\}$, Lemma 2.2 shows that there exist constants $\lambda_{0} \in(0,1)$ and $C_{0}$ such that

$$
\mathbb{E}_{x, y} \lambda_{0}^{-\tilde{\rho}} \leqslant C_{0}(L(x)+L(y)+1) \quad \text { for }(x, y) \in X^{2} .
$$

Define $A=\{(x, y, \theta) \in \widehat{X}:(x, y) \in F$ and $L(x)+L(y)<R\}$ and

$$
\rho\left(\left(x_{n}, y_{n}, \theta_{n}\right)_{n \in \mathbb{N}_{0}}\right)=\inf \left\{n \in \mathbb{N}_{0}:\left(x_{n}, y_{n}, \theta_{n}\right) \in A\right\} .
$$


Slightly abusing notation, we identify $\kappa:(X \times X)^{\infty} \rightarrow \mathbb{N}_{0} \cup\{\infty\}$, defined in A4, with its extension to $\widehat{X}^{\infty}: \kappa\left(\left(x_{n}, y_{n}, \theta_{n}\right)_{n \in \mathbb{N}_{0}}\right)=\kappa\left(\left(x_{n}, y_{n}\right)_{n \in \mathbb{N}_{0}}\right)$. Since $\rho \leqslant \tilde{\rho}+\kappa \circ T_{\tilde{\rho}}$, where $T_{\tilde{\rho}}\left(\left(x_{n}, y_{n}, \theta_{n}\right)_{n \in \mathbb{N}_{0}}\right)=\left(x_{n+\tilde{\rho}}, y_{n+\tilde{\rho}}, \theta_{n+\tilde{\rho}}\right)_{n \in \mathbb{N}_{0}}$, an argument similar to that in the proof of Lemma 2.1 shows that there exists $\lambda \in(0,1)$ such that

$$
\mathbb{E}_{x, y, \theta} \lambda^{-\rho} \leqslant \tilde{C} C_{0}(L(x)+L(y)+1) \quad \text { for } x, y \in X, \theta \in\{0,1\} .
$$

Define $B=\{(x, y, \theta) \in \widehat{X}: \theta=1\}$ and

$$
\epsilon\left(\left(x_{n}, y_{n}, \theta_{n}\right)_{n \in \mathbb{N}_{0}}\right)=\inf \left\{n \geqslant 1:\left(x_{n}, y_{n}, \theta_{n}\right) \notin B\right\} .
$$

From Step II we obtain $\mathbb{P}_{x, y, \theta}(B) \geqslant \frac{1}{2} \delta^{n_{0}}$ for $(x, y, \theta) \in A$. From 2.6 and 2.7 it follows that

$$
\begin{aligned}
& \widehat{\mathbf{B}}_{x, y, \theta}(\epsilon=n)=\int_{\widehat{X}^{n}} \widehat{\mathbf{R}}_{z_{n-1}}(\widehat{X}) \widehat{\mathbf{Q}}_{x, y, \theta}^{0, \ldots, n-1}\left(d z_{0}, \ldots, d z_{n-1}\right) \\
& =\left\|\mathbf{Q}_{\delta_{(x, y)}}^{n-1}\right\|-\left\|\widehat{Q}_{\mathbf{Q}_{\delta_{(x, y)}^{n-1}}}\right\| \leqslant l \int_{X^{2}} \bar{d}(u, v) \mathbf{Q}_{\delta_{(x, y)}^{n-1}}(d u, d v) \\
& \leqslant l \bar{\alpha}^{n-1} \bar{d}(x, y)<\bar{\alpha}^{n-1} l R_{0}
\end{aligned}
$$

whenever $(x, y, \theta) \in A$. Finally, Lemma 2.1 guarantees the existence of constants $\gamma \in(0,1)$ and $C_{1}>0$ such that for

$$
\tau\left(\left(x_{n}, y_{n}, \theta_{n}\right)_{n \in \mathbb{N}_{0}}\right)=\inf \left\{n \geqslant 1: \forall_{k \geqslant n}\left(x_{k}, y_{k}, \theta_{k}\right) \in B\right\}
$$

we have

$$
\mathbb{E}_{x, y, \theta} \gamma^{-\tau} \leqslant C_{1}(L(x)+L(y)+1) \quad \text { for } x, y \in X, \theta \in\{0,1\} .
$$

STEP IV. Define $G_{n / 2}=\left\{t \in\left(X^{2} \times\{0,1\}\right)^{\infty}: \tau(t) \leqslant n / 2\right\}$ and $H_{n / 2}=$ $\left\{t \in\left(X^{2} \times\{0,1\}\right)^{\infty}: \tau(t)>n / 2\right\}$. For every $n \in \mathbb{N}$ we have

$$
\widehat{\mathbf{B}}_{x, y, \theta}^{\infty}=\left.\widehat{\mathbf{B}}_{x, y, \theta}^{\infty}\right|_{G_{n / 2}}+\left.\widehat{\mathbf{B}}_{x, y, \theta}^{\infty}\right|_{H_{n / 2}} \quad \text { for } x, y \in X, \theta \in\{0,1\} .
$$

Fix $\theta=1$ and $(x, y) \in X^{2}$. From the fact that $\|\cdot\|_{\mathrm{FM}} \leqslant\|\cdot\|_{\mathrm{W}}$ it follows that

$$
\begin{aligned}
& \left\|P^{* n} \delta_{x}-P^{* n} \delta_{y}\right\|_{\mathrm{FM}}=\left\|\mathbf{P}_{x}^{n}-\mathbf{P}_{y}^{n}\right\|_{\mathrm{FM}} \\
& =\sup _{f \in \mathcal{F}}\left|\int_{X^{2}}\left(f\left(z_{1}\right)-f\left(z_{2}\right)\right)\left(p r_{n}^{\#} \mathbf{B}_{x, y}^{\infty}\right)\left(d z_{1}, d z_{2}\right)\right| \\
& =\sup _{f \in \mathcal{F}}\left|\int_{X^{2}}\left(f\left(z_{1}\right)-f\left(z_{2}\right)\right)\left(p r_{X^{2}}^{\#} p r_{n}^{\#} \widehat{\mathbf{B}}_{x, y, \theta}^{\infty}\right)\left(d z_{1}, d z_{2}\right)\right| \\
& \leqslant \sup _{f \in \mathcal{W}}\left|\int_{X^{2}}\left(f\left(z_{1}\right)-f\left(z_{2}\right)\right)\left(p r_{X^{2}}^{\#} p r_{n}^{\#}\left(\left.\widehat{\mathbf{B}}_{x, y, \theta}^{\infty}\right|_{G_{n / 2}}\right)\right)\left(d z_{1}, d z_{2}\right)\right|+2 \widehat{\mathbf{B}}_{x, y, \theta}^{\infty}\left(H_{n / 2}\right) .
\end{aligned}
$$


From A2 we obtain

$$
\begin{aligned}
\sup _{\mathcal{W}} \mid \int_{X^{2}} & \left(f\left(z_{1}\right)-f\left(z_{2}\right)\right)\left(p r_{X^{2}}^{\#} p r_{n}^{\#}\left(\left.\widehat{\mathbf{B}}_{x, y, \theta}^{\infty}\right|_{G_{n / 2}}\right)\right)\left(d z_{1}, d z_{2}\right) \mid \\
& \leqslant \int_{X^{2}} d\left(z_{1}, z_{2}\right)\left(p r_{X^{2}}^{\#} p r_{n}^{\#}\left(\left.\widehat{\mathbf{B}}_{x, y, \theta}^{\infty}\right|_{G_{n / 2}}\right)\right)\left(d z_{1}, d z_{2}\right) \\
& \leqslant \alpha^{n / 2} \int_{X^{2}} d\left(z_{1}, z_{2}\right)\left(p r_{X^{2}}^{\#} p r_{n / 2}^{\#}\left(\left.\widehat{\mathbf{B}}_{x, y, \theta}^{\infty}\right|_{G_{n / 2}}\right)\right)\left(d z_{1}, d z_{2}\right) \leqslant \alpha^{n / 2} R .
\end{aligned}
$$

Now Step III and the Chebyshev inequality imply that

$$
\widehat{\mathbf{B}}_{x, y, \theta}^{\infty}\left(H_{n / 2}\right) \leqslant \gamma^{n / 2} C_{1}(L(x)+L(y)+1) \quad \text { for } n \in \mathbb{N} .
$$

Taking $C_{2}=2 C_{1}+R$ and $q=\max \left\{\gamma^{n / 2}, \alpha^{n / 2}\right\}$ we obtain

$$
\left\|P^{* n} \delta_{x}-P^{* n} \delta_{y}\right\|_{\mathrm{FM}} \leqslant \gamma^{n} C_{1}(L(x)+L(y)+1) \quad \text { for } x, y \in X, n \in \mathbb{N} \text {, }
$$

and so

$$
\left\|P^{* n} \mu-P^{* n} \nu\right\|_{\mathrm{FM}} \leqslant \gamma^{n} C_{1}\left(\int_{X} L(x) \mu(d x)+\int_{X} L(y) \nu(d y)+1\right)
$$

for $\mu, \nu \in \mathcal{M}_{1}^{L}(X)$ and $n \in \mathbb{N}$.

STEP V. Observe that Step IV and A1 give

$$
\begin{aligned}
\left\|P^{* n} \delta_{x}-P^{*(n+k)} \delta_{x}\right\|_{\mathrm{FM}} \leqslant \int_{X}\left\|P^{* n} \delta_{x}-P^{* n} \delta_{y}\right\|_{\mathrm{FM}} P^{* k} \delta_{x}(d y) \\
\leqslant q^{n} C_{2} \int_{X}(L(x)+L(y)) P^{* k} \delta_{x}(d y) \leqslant q^{n} C_{3}(1+L(x)),
\end{aligned}
$$

so $\left(P^{* n} \delta_{x}\right)_{n \in \mathbb{N}}$ is a Cauchy sequence for every $x \in X$. Since $\mathcal{M}_{1}(X)$ equipped with the norm $\|\cdot\|_{\mathrm{FM}}$ is complete (see [8]), assumption A0 implies the existence of an invariant measure $\mu_{*}$. Assumption A1 gives $\mu_{*} \in \mathcal{M}_{L}(X)$. Applying inequality 2.11 we obtain (2.4). The observation that $\mathcal{M}_{L}(X)$ is dense in $\mathcal{M}_{1}(X)$ in the total variation norm finishes the proof.

REMARK. In Steps IV and V of the above proof we follow M. Hairer [11].

\section{RANDOM ITERATION OF FUNCTIONS}

Let $(X, d)$ be a Polish space and $(\Theta, \Xi)$ a measurable space with a family $\vartheta_{x} \in$ $\mathcal{M}_{1}(\Theta)$ of distributions on $\Theta$ indexed by $x \in X$. The space $\Theta$ serves as a set of indices for a family $\left\{S_{\theta}: \theta \in \Theta\right\}$ of continuous functions from $X$ into $X$. We assume that $(\theta, x) \mapsto S_{\theta}(x)$ is product measurable. 
In this section we study a stochastically perturbed dynamical system $\left(X_{n}\right)_{n \in \mathbb{N}_{0}}$. Its intuitive description is the following: if $X_{0}$ starts at $x_{0}$, then by choosing $\theta_{0}$ at random from $\vartheta_{x_{0}}$ we define $X_{1}=S_{\theta_{0}}\left(x_{0}\right)$. Having $X_{1}$ we select $\theta_{1}$ according to $\vartheta_{X_{1}}$ and put $X_{2}=S_{\theta_{1}}\left(X_{1}\right)$, and so on. More precisely, the process $\left(X_{n}\right)_{n \in \mathbb{N}_{0}}$ can be written as

$$
X_{n+1}=S_{Y_{n}}\left(X_{n}\right), \quad n=0,1, \ldots,
$$

where $\left(Y_{n}\right)_{n \in \mathbb{N}_{0}}$ is a sequence of random elements defined on a probability space $(\Omega, \Sigma$, prob $)$ with values in $\Theta$ such that

$$
\operatorname{prob}\left(Y_{n} \in B \mid X_{n}=x\right)=\vartheta_{x}(B) \quad \text { for } x \in X, B \in \Xi, n=0,1, \ldots,
$$

and $X_{0}: \Omega \rightarrow X$ is a given random variable. Denoting by $\mu_{n}$ the probability law of $X_{n}$, we will give a recurrence relation between $\mu_{n+1}$ and $\mu_{n}$. To this end fix $f \in B_{b}(X)$ and note that $\mathbb{E} f\left(X_{n+1}\right)=\int_{X} f d \mu_{n+1}$. By 3.1) we have

$$
\int_{A} \vartheta_{x}(B) \mu_{n}(d x)=\operatorname{prob}\left(\left\{Y_{n} \in B\right\} \cap\left\{X_{n} \in A\right\}\right) \quad \text { for } B \in \Xi, A \in \mathcal{B}_{X},
$$

hence

$$
\mathbb{E} f\left(X_{n+1}\right)=\int_{\Omega} f\left(S_{Y_{n}(\omega)}\left(X_{n}(\omega)\right) \operatorname{prob}(d \omega)=\int_{X} \int_{\Theta} f\left(S_{\theta}(x)\right) \vartheta_{x}(d \theta) \mu_{n}(d x) .\right.
$$

Putting $f=\mathbf{1}_{A}, A \in \mathcal{B}_{X}$, we obtain $\mu_{n+1}(A)=P^{*} \mu_{n}(A)$, where

$$
P^{*} \mu(A)=\int_{X} \int_{\Theta} \mathbf{1}_{A}\left(S_{\theta}(x)\right) \vartheta_{x}(d \theta) \mu(d x) \quad \text { for } \mu \in \mathcal{M}_{\text {fin }}(X), A \in \mathcal{B}_{X} .
$$

In other words this formula defines the transition operator for $\mu_{n}$. The operator $P^{*}$ is the adjoint of the Markov operator $P: B_{b}(X) \rightarrow B_{b}(X)$ of the form

$$
P f(x)=\int_{\Theta} f\left(S_{\theta}(x)\right) \vartheta_{x}(d \theta) .
$$

We take this formula as the precise formal definition of the process under study. We will show that the operator (3.2) has a unique invariant measure provided the following conditions hold:

B1. There exists $\alpha \in(0,1)$ such that

$$
\int_{\Theta} d\left(S_{\theta}(x), S_{\theta}(y)\right) \vartheta_{x}(d \theta) \leqslant \alpha d(x, y) \quad \text { for } x, y \in X .
$$

B2. There exists $\bar{x} \in X$ such that

$$
c:=\sup _{x \in X} \int_{\Theta} d\left(S_{\theta}(\bar{x}), \bar{x}\right) \vartheta_{x}(d \theta)<\infty .
$$


B3. The map $x \mapsto \vartheta_{x}, x \in X$, is Hölder continuous in the total variation norm, i.e. there exist $l>0$ and $\nu \in(0,1]$ such that

$$
\left\|\vartheta_{x}-\vartheta_{y}\right\| \leqslant l d(x, y)^{\nu} \quad \text { for } x, y \in X
$$

B4. There exists $\delta>0$ such that

$$
\vartheta_{x} \wedge \vartheta_{y}\left(\left\{\theta \in \Theta: d\left(S_{\theta}(x), S_{\theta}(y)\right) \leqslant \alpha d(x, y)\right\}\right)>\delta \quad \text { for } x, y \in X
$$

where $\wedge$ denotes the greatest lower bound in the lattice of finite measures.

REMARK. In the simplest case $\Theta=\{1, \ldots, n\}$ the above conditions are standard and nearly optimal (see [28] for a discussion).

Proposition 3.1. Assume B1-B4. Then the operator (3.2) has a unique invariant measure $\mu_{*} \in \mathcal{M}_{1}^{1}(X)$, which is attractive in $\mathcal{M}_{1}(X)$. Moreover there exist $q \in(0,1)$ and $C>0$ such that

$$
\left\|P^{* n} \mu-\mu_{*}\right\|_{\mathrm{FM}} \leqslant q^{n} C\left(1+\int_{X} d(\bar{x}, x) \mu(d x)\right)
$$

for $\mu \in \mathcal{M}_{1}^{1}(X)$ and $n \in \mathbb{N}$.

Proof. Define the operator $Q$ on $B_{b}\left(X^{2}\right)$ by

$$
Q(f)(x, y)=\int_{\Theta} f\left(S_{\theta}(x), S_{\theta}(y)\right) \vartheta_{x} \wedge \vartheta_{y}(d \theta) .
$$

Since

$$
\left\|\vartheta_{x^{\prime}} \wedge \vartheta_{y^{\prime}}-\vartheta_{x} \wedge \vartheta_{y}\right\| \leqslant 2\left(\left\|\vartheta_{x^{\prime}}-\vartheta_{x}\right\|+\left\|\vartheta_{y^{\prime}}-\vartheta_{y}\right\|\right)
$$

it follows that

$$
\begin{aligned}
\left|Q(f)\left(x^{\prime}, y^{\prime}\right)-Q(f)(x, y)\right| \leqslant \int_{\Theta}\left|f\left(S_{\theta}\left(x^{\prime}\right), S_{\theta}\left(y^{\prime}\right)\right)\right|\left\|\vartheta_{x^{\prime}} \wedge \vartheta_{y^{\prime}}-\vartheta_{x} \wedge \vartheta_{y}\right\|(d \theta) \\
\quad+\int_{\Theta}\left|f\left(S_{\theta}\left(x^{\prime}\right), S_{\theta}\left(y^{\prime}\right)\right)-f\left(S_{\theta}(x), S_{\theta}(y)\right)\right| \vartheta_{x} \wedge \vartheta_{y}(d \theta) \\
\leqslant 2 l \sup _{z \in X^{2}}|f(z)|\left(d\left(x, x^{\prime}\right)^{\nu}+d\left(y, y^{\prime}\right)^{\nu}\right) \\
\quad+\int_{\Theta}\left|f\left(S_{\theta}\left(x^{\prime}\right), S_{\theta}\left(y^{\prime}\right)\right)-f\left(S_{\theta}(x), S_{\theta}(y)\right)\right| \vartheta_{x} \wedge \vartheta_{y}(d \theta),
\end{aligned}
$$

for $f \in B_{b}\left(X^{2}\right)$ and $x, y \in X$. Consequently, $Q\left(C_{b}\left(X^{2}\right)\right) \subset C_{b}\left(X^{2}\right)$, by Lebesgue's dominated convergence theorem. Put

$$
\mathcal{F}=\left\{f \in B_{b}\left(X^{2}\right): \sup _{z \in X^{2}}|f(z)| \leqslant M, Q(f) \in B_{b}\left(X^{2}\right)\right\},
$$


where $M>0$ is fixed, and observe that the family $\mathcal{F}$ contains all continuous functions bounded by $M$ and the limit of any convergent sequence of functions in the class, i.e. $\mathcal{F}$ consists, by definition, of all Baire functions bounded by $M$. Since the class of Baire functions is identical with the class of Borel functions (see [22, Theorem 4.5.2]) it follows that $Q\left(B_{b}\left(X^{2}\right)\right) \subset B_{b}\left(X^{2}\right)$. In particular, for the family $\left\{Q_{x, y}: x, y \in X\right\}$ of (subprobability) measures given by

$$
Q_{x, y}(C)=\int_{\Theta} \mathbf{1}_{C}\left(S_{\theta}(x), S_{\theta}(y)\right) \vartheta_{x} \wedge \vartheta_{y}(d \theta),
$$

the maps $(x, y) \mapsto Q_{x, y}(C)$ are measurable for every $C \in \mathcal{B}_{X^{2}}$.

Arguing similarly we show that (3.2) is a well defined Feller operator. It has Lyapunov function $L(x)=d(x, \bar{x})$, since $\int_{\Theta} d\left(S_{\theta}(x), \bar{x}\right) \vartheta_{x}(d \theta) \leqslant \alpha d(x, \bar{x})+c$. Now, observe that

$$
\begin{aligned}
\left\|Q_{x, y}\right\| & =\vartheta_{x} \wedge \vartheta_{y}(\Theta)=\inf _{A \in \Xi}\left\{\vartheta_{x}(A)+\vartheta_{y}(\Theta \backslash A)\right\} \\
& =1-\sup _{A \in \Xi}\left\{\vartheta_{y}(A)-\vartheta_{x}(A)\right\}=1-\left\|\vartheta_{x}-\vartheta_{y}\right\| \geqslant 1-l d(x, y)^{\nu}
\end{aligned}
$$

for $x, y \in X$. Moreover,

$$
\int_{X^{2}} d(u, v) Q_{x, y}(d u, d v)=\int_{\Theta} d\left(S_{\theta}(x), S_{\theta}(y)\right) \vartheta_{x} \wedge \vartheta_{y}(d \theta) \leqslant \alpha d(x, y),
$$

and $Q_{x, y}\left(D_{\alpha d(x, y)}\right)=\vartheta_{x} \wedge \vartheta_{y}\left(\left\{\theta \in \Theta: d\left(S_{\theta}(x), S_{\theta}(y)\right) \leqslant \alpha d(x, y)\right\}\right)>\delta$ for $x, y \in X$. In consequence, $\mathbf{A 0} \mathbf{- A 3}$ are fulfilled. The use of Theorem 2.1 (see also comments in Subsection 2.2 concerning assumption A4) ends the proof.

\section{PERPETUITIES WITH PLACE DEPENDENT PROBABILITIES}

Let $X=\mathbb{R}^{d}$ and $G=\mathbb{R}^{d \times d} \times \mathbb{R}^{d}$, and consider the function $S_{\theta}: X \rightarrow X$ defined by $S_{\theta}(x)=M(\theta) x+Q(\theta)$, where $(M, Q)$ is a random variable on $(\Theta, \Xi)$ with values in $G$. Then (3.2) may be written as

$$
\operatorname{Pf}(x)=\int_{G} f(m x+q) d \vartheta_{x} \circ(M, Q)^{-1}(m, q) .
$$

This operator is connected with the random difference equation of the form

$$
\Phi_{n}=M_{n} \Phi_{n-1}+Q_{n}, \quad n=1,2, \ldots,
$$

where $\left(M_{n}, Q_{n}\right)_{n \in \mathbb{N}}$ is a sequence of independent random variables distributed as $(M, Q)$. Namely, the process $\left(\Phi_{n}\right)_{n \in \mathbb{N}_{0}}$ is a homogeneous Markov chain with transition kernel $P$ given by

$$
P f(x)=\int_{G} f(m x+q) d \mu(m, q),
$$

where $\mu$ stands for the distribution of $(M, Q)$. Equation (4.2) arises in various areas including economics, physics, nuclear technology, biology and sociology (see 
e.g. [32]). It is closely related to a sequence $\left(\Psi_{n}\right)_{n \in \mathbb{N}}$ of backward iterations, given by $\sum_{k=1}^{n} M_{1} \ldots M_{k-1} Q_{k}, n \in \mathbb{N}$ (see e.g. [9]). Under conditions ensuring the almost sure convergence of the sequence $\left(\Psi_{n}\right)_{n \in \mathbb{N}}$ the limiting random variable

$$
\sum_{n=1}^{\infty} M_{1} \ldots M_{n-1} Q_{n}
$$

is often called a perpetuity. It turns out that the probability law of (4.4) is a unique invariant measure for (4.3). The name perpetuity comes from perpetual payment streams and recently gained some popularity in the literature on stochastic recurrence equations (see [7]). In the insurance context a perpetuity represents the present value of a permanent commitment to make a payment at regular intervals, say annually, into the future forever. The $Q_{n}$ represent annual payments, and the $M_{n}$ cumulative discount factors. Many interesting examples of perpetuities can be found in [1]. Due to the significant papers [17], [10], [32] and [9] we have a complete (in dimension one) characterization of convergence of perpetuities. The rate of that convergence has recently been extensively studied by many authors (see for instance [3]-[5], [24]). The main result of this section concerns the rate of convergence of the process $\left(X_{n}\right)_{n \in \mathbb{N}_{0}}$ associated with the operator $P: B_{b}\left(\mathbb{R}^{d}\right) \rightarrow B_{b}\left(\mathbb{R}^{d}\right)$ given by

$$
\operatorname{Pf}(x)=\int_{G} f(m x+q) d \mu_{x}(m, q),
$$

where $\left\{\mu_{x}: x \in \mathbb{R}^{d}\right\}$ is a family of Borel probability measures on $G$. In contrast to $\left(\Phi_{n}\right)_{n \in \mathbb{N}_{0}}$, the process $\left(X_{n}\right)_{n \in \mathbb{N}_{0}}$ moves by choosing $\theta$ at random from a measure depending on $x$. Taking into consideration the concept of perpetuity we may say that $\left(X_{n}\right)_{n \in \mathbb{N}_{0}}$ forms a perpetuity with place dependent probabilities.

COROLlARY 4.1. Assume that $\left\{\mu_{x}: x \in \mathbb{R}^{d}\right\}$ is a family of Borel probability measures on $G$ such that

$$
\alpha:=\sup _{x \in \mathbb{R}^{d}} \int_{G}\|m\|_{\mathrm{op}} d \mu_{x}(m, q)<1, \quad c:=\sup _{x \in \mathbb{R}^{d}} \int_{G}|q| d \mu_{x}(m, q)<\infty .
$$

Assume moreover that the map $x \mapsto \mu_{x}, x \in X$, is Hölder continuous in the total variation norm and there exists $\delta>0$ such that

$$
\mu_{x} \wedge \mu_{y}\left(\left\{(m, q) \in G:\|m\|_{\text {op }} \leqslant \alpha\right\}\right)>\delta \quad \text { for } x, y \in \mathbb{R}^{d} .
$$

Then the operator 4.5 has a unique invariant measure $\mu_{*} \in \mathcal{M}_{1}^{1}\left(\mathbb{R}^{d}\right)$, which is attractive in $\mathcal{M}_{1}\left(\mathbb{R}^{d}\right)$. Moreover there exist $q \in(0,1)$ and $C>0$ such that

$$
\left\|P^{* n} \mu-\mu_{*}\right\|_{\mathrm{FM}} \leqslant q^{n} C\left(1+\int_{\mathbb{R}^{d}}|x| \mu(d x)\right)
$$

for $\mu \in \mathcal{M}_{1}^{1}\left(\mathbb{R}^{d}\right)$ and $n \in \mathbb{N}$.

The proof of the corollary is a straightforward application of Proposition 3.1 We leave the details to the reader. 
We finish the paper by giving an example illustrating Corollary 4.1 .

EXAMPLE. Let $\nu_{0}, \nu_{1}$ be distributions on $\mathbb{R}^{2}$. Assume that $p, q: \mathbb{R} \rightarrow[0,1]$ are Lipschitz functions (with Lipschitz constant $L$ ) summing to 1 , and $p(x)=1$ for $x \leqslant 0$, while $p(x)=0$ for $x \geqslant 1$. Define $\mu_{x}$ by $\mu_{x}=p(x) \nu_{0}+q(x) \nu_{1}, x \in \mathbb{R}$. Then:

- $\left\|\mu_{x}-\mu_{y}\right\| \leqslant 2 L|x-y|$ for $x, y \in \mathbb{R}$.

- If $\int_{\mathbb{R}^{2}}|m| d \nu_{i}(m, q)<1$ and $\int_{\mathbb{R}^{2}}|q| d \nu_{i}(m, q)<\infty$ for $i=0,1$, then 4.6 holds.

- For all $A \in \mathcal{B}_{\mathbb{R}^{2}}$ and $x, y \in \mathbb{R}$ we have

$$
\begin{aligned}
\mu_{x} \wedge \mu_{y}(A) & \geqslant \nu_{0} \wedge \nu_{1}(A)=\left(\nu_{0}-\lambda^{+}\right)(A)=\left(\nu_{1}-\lambda^{-}\right)(A) \\
& \geqslant \max \left\{\nu_{0}(A), \nu_{1}(A)\right\}-\left\|\nu_{0}-\nu_{1}\right\|(A),
\end{aligned}
$$

where $\left(\lambda^{+}, \lambda^{-}\right)$is the Jordan decomposition of $\nu_{1}-\nu_{0}$.

Acknowledgments. This work was partially supported by the Faculty of Applied Mathematics AGH UST statutory tasks within a subsidy of Ministry of Science and Higher Education of Poland.

\section{REFERENCES}

[1] G. Alsmeyer, A. Iksanov and U. Rösler, On distributional properties of perpetuities, J. Theoret. Probab. 22 (2009), 666-682.

[2] M. F. Barnsley, S. G. Demko, J. H. Elton and J. S. Geronimo, Invariant measures for Markov processes arising from iterated function systems with place dependent probabilities, Ann. Inst. H. Poincaré 24 (1988), 367-394.

[3] K. Bartkiewicz, A. Jakubowski, T. Mikosch and O. Wintenberger, Stable limits for sums of dependent infinite variance random variables, Probab. Theory Related Fields 150 (2011), 337372.

[4] S. Brofferio, D. Buraczewski and E. Damek, On the invariant measure of the random difference equation $X_{n}=A_{n} X_{n-1}+B_{n}$ in the critical case, Ann. Inst. H. Poincaré Probab. Statist. 48 (2012), 377-395.

[5] D. Buraczewski, E. Damek and Y. Guivarc'h, Convergence to stable laws for a class of multidimensional stochastic recursions, Probab. Theory Related Fields 148 (2010), 333-402.

[6] P. Diaconis and D. Freedman, Iterated random functions, SIAM Rev. 41 (1999), 45-76.

[7] P. Embrechts, C. Klüppelberg and T. Mikosch, Modeling Extremal Events for Insurance and Finance, Appl. Math. 33, Springer, New York, 1997.

[8] S. Ethier and T. Kurtz, Markov Processes, Wiley, New York, 1986.

[9] C. M. Goldie and R. A. Maller, Stability of perpetuities, Ann. Probab. 28 (2000), 1195-1218.

[10] A. K. Grincevičjus, On the continuity of the distribution of a sum of dependent variables connected with independent walks on lines, Theory Probab. Appl. 19 (1974), 163-168.

[11] M. Hairer, Exponential mixing properties of stochastic PDEs through asymptotic coupling, Probab. Theory Related Fields 124 (2002), 345-380.

[12] M. Hairer and J. Mattingly, Spectral gaps in Wasserstein distances and the 2D stochastic Navier-Stokes equations, Ann. Probab. 36 (2008), 2050-2091.

[13] M. Hairer, J. Mattingly and M. Scheutzow, Asymptotic coupling and a general form of Harris' theorem with applications to stochastic delay equations, Probab. Theory Related Fields 149 (2011), 223-259. 
[14] S. Hille, K. Horbacz, T. Szarek and H. Wojewódka, Limit theorems for some Markov chains, J. Math. Anal. Appl. 443 (2016), 385-408.

[15] K. Horbacz and T. Szarek, Continuous iterated function systems on Polish spaces, Bull. Polish Acad. Sci. Math. 49 (2001), 191-202.

[16] K. Horbacz, The central limit theorem for random dynamical systems, J. Statist. Phys. 164 (2016), 1261-1291.

[17] H. Kesten, Random difference equations and renewal theory for products of random matrices, Acta Math. 131 (1973), 207-248.

[18] T. Komorowski and A. Walczuk, Central limit theorem for Markov processes with spectral gap in the Wasserstein metric, Stoch. Process. Appl. 122 (2012), 2155-2184.

[19] S. Kuksin and A. Shirikyan, A coupling approach to randomly forced nonlinear PDE's. I, Comm. Math. Phys. 221 (2001), 351-366.

[20] S. Kuksin, A. Piatnitsky and A. Shirikyan, A coupling approach to randomly forced nonlinear PDE's. II, Comm. Math. Phys. 230 (2002), 81-85.

[21] T. Lindvall, Lectures on the Coupling Method, Wiley, New York, 1992.

[22] S. Łojasiewicz, An Introduction to the Theory of Real Function, Wiley, Chichester, 1998.

[23] S. Meyn and R. L. Tweedie, Markov Chains and Stochastic Stability, 2nd ed., Cambridge Univ. Press, Cambridge, 2009.

[24] M. Mirek, Heavy tail phenomenon and convergence to stable laws for iterated Lipschitz maps, Probab. Theory Related Fields 151 (2011), 705-734.

[25] A. Öberg, Approximation of invariant measures for random iterations, Rocky Mountain J. Math. 36 (2006), 273-301.

[26] C. Odasso, Exponential mixing for stochastic PDEs: the non-additive case, Probab. Theory Related Fields 140 (2008), 41-82.

[27] A. Shirikyan, A version of the law of large numbers and applications, in: Probabilistic Methods in Fluids (Swansea, 2002), World Sci., 2003, 263-271.

[28] M. Ślęczka, The rate of convergence for iterated function systems, Studia Math. 205 (2011), 201-214.

[29] M. Ślęczka, Exponential convergence for Markov systems, Ann. Math. Silesianae 29 (2015), 139-149.

[30] Ö. Stenflo, A note on a theorem of Karlin, Statist. Probab. Lett. 54 (2001), 183-187.

[31] T. Szarek, Invariant measures for nonexpansive Markov operators on Polish spaces, Dissertationes Math. 415 (2003), 62 pp.

[32] W. Vervaat, On a stochastic difference equation and a representation of non-negative infinitely divisible random variables, Adv. Appl. Probab. 11 (1979), 750-783.

[33] I. Werner, Contractive Markov sysems, J. London Math. Soc. 71 (2005), 236-258.

[34] L. Xu, Exponential mixing of 2D SDEs forced by degenerate Levy noises, J. Evolutionary Equations 14 (2014), 249-272.

Rafał Kapica

Faculty of Applied Mathematics

AGH University of Science and Technology

al. A. Mickiewicza 30

30-059 Kraków, Poland

E-mail: rafal.kapica@agh.edu.pl
Maciej Ślęczka

Institute of Mathematics University of Silesia in Katowice

Bankowa 14

40-007 Katowice, Poland

E-mail: sleczka@math.us.edu.pl

Received 10.11.2017;

revised version 8.1 .2019 
